\title{
CHARACTERIZATIONS OF ULTRADISTRIBUTIONS WITH COMPACT SUPPORT AND DECOMPOSITION BY SUPPORT
}

\author{
SOON-YEONG CHUNG AND DOHAN KIM
}

(Communicated by Palle E. T. Jorgensen)

\begin{abstract}
In this paper we prove that every nonquasianalytic ultradistribution can be uniformly majorized by the behavior of test functions only on the support and that every ultradistribution with support in the union $K_{1} \cup K_{2}$ of two compact sets can be decomposed as the sum of one with support in $K_{1}$ and one with support in $K_{2}$, along the context of Malgrange [17].
\end{abstract}

\section{INTRODUCTION AND PRELIMINARIES}

Let $M=\left(M_{p}\right)$ be a sequence of positive numbers satisfying

(i) $M_{0}=1$,

(ii) $M_{p}^{2} \leq M_{p-1} M_{p+1}, p=1,2, \ldots$,

(iii) $\sum_{p=1}^{\infty} M_{p-1} / M_{p}<+\infty$.

If $\Omega \subset R^{n}$ is an open set we shall denote by $C_{M}(\Omega)$ the set of all $\phi \in C^{\infty}(\Omega)$ such that for every compact set $K \subset \Omega$ there exist constants $h=h_{K}>0$ and $C=C_{K, h}>0$ such that

$$
\sup _{x \in K}\left|D^{\alpha} \phi(x)\right| \leq C h^{|\alpha|} M_{|\alpha|}
$$

for any multi-index $\alpha \in N^{n}$. Here we say that such a $\phi$ is ultradifferentiable in $\Omega$. Condition (iii) above guarantees that we can construct cutoff functions and partitions of unity from the Denjoy-Carleman theorem. The class is then called nonquasianalytic. In particular when $M_{p}=(p !)^{s}$ for $s>1$, the class $C_{M}$ is Gevrey class of order $s$. Let $C_{M}(K, h)$ be the set of all $\phi \in C^{\infty}(\Omega)$ with

$$
|\phi|_{K, h}=\sup _{\substack{x \in K \\ \alpha \in N^{n}}} \frac{\left|D^{\alpha} \phi(x)\right|}{h^{|\alpha|} M_{|\alpha|}}<+\infty .
$$

Received by the editors May 15, 1989.

1980 Mathematics Subject Classification (1985 Revision). Primary 46F05, 46F15.

Key words and phrases. Nonquasianalyticity, ultradistribution, DF-space.

The first author was partially supported by Korea Research Foundation and the second author was partially supported by KOSEF. 
Then $C_{M}(K, h)$ with the norm $|\cdot|_{K, h}$ is a Banach space and

$$
C_{M}(\Omega)=\bigcap_{K \Subset \Omega} \bigcup_{h>0} C_{M}(K, h) .
$$

The topology on $C_{M}(\Omega)$ is $\lim _{K \in \Omega}{\underset{\lim }{\longleftrightarrow} \rightarrow \infty}_{h \rightarrow \infty} C_{M}(K, h)$ where $\stackrel{\text { lim }}{\longleftarrow}$ and

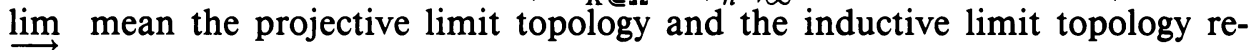
spectively.

Let $K \subset R^{n}$ be a compact set. The space $\mathscr{E}_{M}^{\prime}(K)$ of ultradistributions with support in $K$ is defined to be the set of all continuous linear functionals on $C_{M}\left(R^{n}\right)$ such that for every neighborhood $\Omega$ of $K$ and for every $h>0$ we have

$$
|u(\phi)| \leq C|\phi|_{\Omega, h}, \quad \phi \in C_{M}(\bar{\Omega}, h)
$$

for some constant $C=C_{\Omega, h}>0$.

In (1.4) if we replace $\Omega$ by $K$ then the choice of the constant $C$ may not be uniform. In other words, $u(\phi)$ cannot be uniformly majorized by the behavior of $\phi$ on $K$. In this paper, by obtaining an alternative description of $\mathscr{E}_{M}^{\prime}(K)$, it will be shown that $u(\phi)$ can be estimated by the behavior of $\phi$ only on $K$. The space $\mathscr{E}_{M}^{\prime}(K)$ is characterized in several ways in $\S 2$.

In $\S 3$ we examine whether every $u \in \mathscr{E}_{M}^{\prime}\left(K_{1} \cup K_{2}\right)$ can be written as $u=$ $u_{1}+u_{2}, u_{j} \in \mathscr{E}_{M}^{\prime}\left(K_{j}\right), j=1,2$. This is possible positively for hyperfunctions and quasianalytic ultradistributions (see Hörmander [11, 12]) but, in general, not true for the Schwartz distributions and nonquasianalytic ultradistributions (Lojasiewicz [16], Hörmander [11]). Lojasiewicz [16] gave the geometric condition for $K_{1}$ and $K_{2}$ that such a decomposition is possible. Here we give a necessary and sufficient condition that such a decomposition theorem holds for the nonquasianalytic ultradistributions, which will be verified along the context of Malgrange [17].

\section{THE CHARACTERIZATIONS OF $\mathscr{E}_{M}^{\prime}(K)$}

Let $M=\left(M_{p}\right)$ be a sequence of positive numbers satisfying, in addition to (1.1), that for some constant $H>1$

$$
\begin{aligned}
& \text { (i) } M_{p+1} \leq H^{p+q} M_{p} M_{q}, p, q=1,2, \ldots, \\
& \text { (ii) } M_{p+1}^{p} \leq H^{p} M_{p}^{p+1}, p=0,1,2, \ldots, \\
& \text { (iii) } M_{p} / p M_{p-1} \text { is increasing, } \\
& \text { (iv) } \sum_{q=p+1}^{\infty} M_{q-1} / M_{q} \leq H^{p} M_{p} / M_{p+1}, p=1,2, \ldots
\end{aligned}
$$

Let $K$ be a compact set in $R^{n}$. A jet in $K$ is a multisequence $F=\left(f^{k}\right)$ of continuous functions on $K$. For a jet, for $x, y \in K, m \in N$ we put

$$
\left(R_{y}^{m} F\right)^{k}(x)=f^{k}(x)-\sum_{|l| \leq m-|k|} f^{k+l}(y) \frac{(x-y)^{l}}{l !} .
$$


It is clear that if $f \in C_{M}\left(R^{n}\right)$, the jet $F=\left(f^{k}\right)$, with $f^{k}=D^{k} f$, satisfies the conditions

$$
\begin{gathered}
\left|f^{k}(x)\right| \leq C h^{|k|} M_{|k|}, \quad k \in N^{n}, \quad x \in K, \\
\left|\left(R_{y}^{m} F\right)^{k}(x)\right| \leq \frac{C|x-y|^{m-|k|+1} h^{m+1} M_{m+1}}{(m-|k|+1) !}, \\
\quad x, y \in K, m \in N,|k| \leq m,
\end{gathered}
$$

for some constants $C>0$ and $h>0$.

Definition 2.1. A jet $F=\left(f^{k}\right)$ on $K$ is called a (Whitney) jet of class $M$ if it satisfies conditions (2.2) and (2.3). We write $\mathscr{E}_{M}(K)$ for the space of Whitney jets of class $M$ on $K$.

We need the following lemma later in this paper; (given in Bruna [3]). It is an extension theorem of Whitney type.

Lemma 2.2. For any $F \in \mathscr{E}_{M}(K)$ there exists $\tilde{f} \in C_{M}\left(R^{n}\right)$ such that $D^{k} \tilde{f}(x)=$ $f^{k}(x)$ for all $x \in K$ and $k \in N^{n}$.

We denote by $\mathscr{E}_{M}(K, h)$ the space of jet $F=\left(f^{k}\right)$ of class of $M$ on $K$ such that

$$
\|F\|_{K, h}=\sup _{\substack{x \in K \\ \alpha \in N^{n}}} \frac{\left|f^{\alpha}(x)\right|}{h^{|\alpha|} M_{|\alpha|}}+\sup _{\substack{x \neq y \in K \\ m \in N,|k| \leq m}} \frac{(m-|k|+1) !\left|\left(R_{y}^{m} F\right)^{k}(x)\right|}{|x-y|^{m-|k|+1} h^{m+1} M_{m+1}}
$$

is finite. Then $\mathscr{E}_{M}(K, h)$ is a Banach space with compact imbedding $\mathscr{E}_{M}(K, h)$ $\hookrightarrow \mathscr{E}_{M}\left(K, h^{\prime}\right)$ when $h<h^{\prime}$. Then the space $\mathscr{E}_{M}(K)$ is $\bigcup_{h>0} \mathscr{E}_{M}(K, h)$ and the topology on $\mathscr{E}_{M}(K)$ is given by the inductive limit ${\underset{\lim }{\longrightarrow}}_{h \rightarrow \infty} \mathscr{E}_{M}(K, h)$.

It follows from Lemma 2.2 that we may consider the space $\mathscr{E}_{M}(K)$ as the set of jets $\left(D^{k} f\right)_{k \in N^{n}}$ where $f \in C_{M}\left(R^{n}\right)$. From this point of view, we have the following lemma.

Lemma 2.3. Let $K$ be a compact convex set in $R^{n}$. Then the topologies on $\mathscr{E}_{M}(K)$ given by the norm $\|\cdot\|_{K, h}$ and the norm $|\cdot|_{K, h}$ are equivalent.

Proof. First, it follows from the definition that

$$
\|F\|_{K, h} \geq|F|_{K, h} \text {. }
$$

On the other hand, Taylor's formula implies that there is $z \in K$ such that

$$
\begin{aligned}
\left|\left(R_{y}^{m} F\right)^{k}(x)\right| & =\left|\sum_{|l|=m-|k|+1} f^{k+l}(z) \frac{(y-x)^{l}}{l !}\right| \\
& \leq \sum_{|l|=m-|k|+1}|F|_{K, h^{\prime}} h^{m+1} M_{m+1} \frac{|x-y|^{m-|k|+1}}{l !} \\
& \leq \frac{|F|_{K, h}(n h)^{m+1} M_{m+1}|x-y|^{m-|k|+1}}{(m-|k|+1) !} .
\end{aligned}
$$


Then it follows that

$$
\|F\|_{K, n h} \leq 2|F|_{K, h}
$$

which completes the proof.

Now we define an ideal of $C_{M}(\Omega)$ as follows:

$$
J_{M}(K: \Omega)=\left\{\phi \in C_{M}(\Omega)\left|D^{\alpha} \phi\right|_{K}=0 \text { for all } \alpha\right\} .
$$

Lemma 2.4. $J_{M}(K: \Omega)$ is the closure of the set of all functions in $C_{M}(\Omega)$ vanishing in a neighborhood of $K$.

Proof. The proof of the closedness is trivial. Now let $\phi$ be an element in $J_{M}(K: \Omega)$ and $\varepsilon$ arbitrary. Choose a function $\theta(x)$ in $C_{M}\left(R^{n}\right)$ such that

$$
\theta(x)= \begin{cases}1 & \text { if } x \in R^{n} \backslash K_{\varepsilon}, \\ 0 & \text { if } x \in K_{\varepsilon / 2},\end{cases}
$$

where $K_{\varepsilon}=\left\{x \in R^{n} \mid d(x, K)<\varepsilon\right\}$. Then $\theta \phi$ vanishes in $K_{\varepsilon / 2}$. It follows from Taylor's formula and (2.1) that for some $C$ and $h$,

$$
\begin{aligned}
& \left|D^{\alpha} \phi(x)\right| \leq C h^{|\alpha|} M_{|\alpha|} d(x, K), \\
& \left|D^{\alpha}(\theta \phi)\right| \leq C h^{|\alpha|} M_{|\alpha|} d(x, K),
\end{aligned}
$$

for all $x \in \Omega$ and $\alpha \in N^{n}$. Then for any compact set $K^{\prime}$ in $\Omega$,

$$
\sup _{\substack{x \in K^{\prime} \\ \alpha \in N^{n}}} \frac{\left|D^{\alpha}(\phi-\theta \phi)\right|}{h^{|\alpha|} M_{|\alpha|}} \leq 2 C \varepsilon,
$$

which completes the proof.

Now consider a map $r: C_{M}\left(R^{n}\right) \rightarrow \mathscr{E}_{M}(K)$ with $r(\phi)=\left(\left.D^{\alpha} \phi\right|_{K}\right)$. Then $r$ is surjective by Lemma 2.2 and we obtain from (2.6) that

$$
\|r(\phi)\|_{K, n h} \leq 2|\phi|_{\widehat{K}, h}
$$

where $\hat{K}$ is the convex hull of $K$. Thus $r$ is a continuous surjection. From this property and Lemma 2.4 we have the following theorem.

Theorem 2.5. The sequence $0 \rightarrow J_{M}\left(K: R^{n}\right) \hookrightarrow C_{M}\left(R^{n}\right) \stackrel{r}{\rightarrow} \mathscr{E}_{M}(K) \rightarrow 0$ is topologically exact.

This theorem implies that $\mathscr{E}_{M}(K)$ is topologically isomorphic to $C_{M}\left(R^{n}\right) /$ $J\left(K: R^{n}\right)$. Thus a continuous linear functional $u$ on $\mathscr{E}_{M}(K)$ is an ultradistribution which is orthogonal to $J_{M}\left(K: R^{n}\right)$, so that it defines an ultradistribution with support in $K$. It follows from Lemma 2.4 that $\mathscr{E}_{M}^{\prime}(K)$ is the space of all ultradistributions with support in $K$. Thus the following is an alternative description of $\mathscr{E}_{M}^{\prime}(K)$ which was defined in $\S 1$. 
Corollary 2.6. We have $u \in \mathscr{E}_{M}^{\prime}(K)$ if and only if for any $h>0$ there exists a constant $C$ such that

$$
|u(\phi)| \leq C\left[\sup _{\substack{x \in K \\ \alpha \in N^{n}}} \frac{\left|D^{\alpha} \phi\right|}{h^{|\alpha|} M_{|\alpha|}}+\sup _{\substack{x \neq y \in K \\ m \in N|k| \leq m}} \frac{(m-|k|+1) !\left|\left(R_{y}^{m} F\right)^{k}(x)\right|}{|x-y|^{m-|k|+1} h^{m+1} M_{m+1}}\right] .
$$

Corollary 2.7. If $u \in \mathscr{E}_{M}^{\prime}(K)$ and $D^{k} \phi(x)=0$ for all $\alpha$ and $x \in K$ then $u(\phi)=0$.

The following example shows that the right-hand side of (2.11) cannot be replaced by $|\phi|_{K, h}$ simply.

Example 2.8. Let $\left(x_{j}\right)$ be a sequence in $R^{n}$ such that

$$
\left|x_{1}\right|>\left|x_{2}\right|>\cdots \rightarrow 0 \text {. }
$$

Let $K$ be a compact set consisting of the sequence $\left(x_{j}\right)$ and its limit 0 . Define

$$
u(\phi)=\sum_{j} m_{j}\left[\phi\left(x_{j}\right)-\phi(0)\right],
$$

where $\left(m_{j}\right)$ is a positive sequence such that

$$
\sum_{j} m_{j}\left|x_{j}\right|=1, \quad \sum_{j} m_{j}=\infty .
$$

Such a sequence always exists since $\lim \left|x_{j}\right|=0$. Then $u$ defines an ultradistribution with support $K$; in fact, it defines a distribution of order 1 with support $K$.

Now we suppose that the following is true: for every $h>0$, there is a constant $C>0$ such that $|u(\phi)| \leq C|\phi|_{K, h}, \phi \in C_{M}\left(R^{n}\right)$.

Choose $\phi \in C_{M}\left(R^{n}\right)$ equal to 1 in a neighborhood of $\left\{x_{1}, \ldots, x_{j}\right\}$ and 0 near $\left\{x_{j+1}, x_{j+2}, \ldots\right\} \cup\{0\}$. Then it follows that

$$
\sum_{i \leq j} m_{i} \leq C,
$$

which gives a contradiction when $j \rightarrow \infty$.

On the other hand, even if the second term of the right-hand side in (2.11) cannot be omitted, we give an optimal condition for this.

Theorem 2.9. Let $K$ be a compact set in $R^{n}$ with finitely many connected components such that any two points $x, y$ in the same component can be joined by a rectifiable curve in $K$ of length $\leq B|x-y|$. If $u$ is an ultradistribution with support in $K$, it follows that for any $h>0$ there exists a constant $C>0$ such that

$$
|u(\phi)| \leq C \sup _{\substack{x \in K \\ \alpha \in N^{n}}} \frac{\left|D^{\alpha} \phi(x)\right|}{h^{|\alpha|} M_{|\alpha|}}, \quad \phi \in C_{M} .
$$


Proof. Let $s \rightarrow x(s)$ be a curve in $K$ with $x(0)=y$ and arclength $s$. Then

$$
\left|F_{k}(s)\right| \leq \frac{C s^{m-|k|+1}}{(m-|k|+1) !} \sum_{|l|=m+1} \sup _{x \in K}\left|D^{l} \phi(x)\right|
$$

if

$$
F_{k}(s)=D^{k} \phi(x(s))-\sum_{|l| \leq m-|k|} D^{k+l} \phi(y) \frac{(x(s)-y)^{l}}{l !} .
$$

This is obvious when $|k|=m$. If $|k|<m$ and (2.13) is already proved for derivatives of higher order, we conclude by induction that

$$
\left|\frac{d F_{k}(s)}{d s}\right| \leq \frac{C s^{m-|k|}}{(m-|k|) !} \sum_{|l|=m+1} \sup _{x \in K}\left|D^{l} \phi\right| .
$$

Since $F_{k}(0)=0$ we obtain (2.13) with $C$ replaced by $n C$. If $d(x, y)$ is the infimum of the curves from $x$ to $y$ in $K$ then (2.13) gives

$$
\begin{aligned}
& \left|D^{k} \phi(x)-\sum_{|l| \leq m-|k|} D^{k+l} \phi(y) \frac{(y-x)^{l}}{l !}\right| \\
& \quad \leq \frac{C d(x, y)^{m-|k|+1}}{(m-|k|+1) !} \sum_{|l| \leq m+1} \sup _{x \in K}\left|D^{l} \phi(x)\right| \\
& \quad \leq \frac{C|x-y|^{m-|k l|+1}}{(m-|k|+1) !}(n h B)^{m+1} M_{m+1}|\phi|_{K, h} .
\end{aligned}
$$

Then it follows that

$$
\|\phi\|_{K, A} \leq 2|\phi|_{K, h},
$$

where $A=(n h B)$, which completes the proof.

The sets satisfying the hypotheses in Theorem 2.9 are sometimes said to be regular in the sense of Whitney.

Corollary 2.10. Let $K$ be a regular compact in $R^{n}$ and $\phi$ a function in $C^{\infty}(K)$ with $|\phi|_{K, h}<+\infty$. Then there exists an extension $\tilde{\phi} \in C_{M}\left(R^{n}\right)$ of $\phi$.

Proof. We use (2.14) combined with Lemma 2.2. Then it is obvious.

\section{DECOMPOSITION THEOREM}

Let $u$ be an ultradistribution with support in the union $K_{1}$ and $K_{2}$ of two compact sets $K_{1}, K_{2}$. In 1985, Hörmander [11] has given an example showing that $u$ cannot always be decomposed as the sum of $u_{1} \in \mathscr{E}_{M}^{\prime}\left(K_{1}\right)$ and $u_{2} \in \mathscr{E}_{M}^{\prime}\left(K_{2}\right)$. In this section we give a necessary and sufficient condition for $K_{1}$ and $K_{2}$ so that such a decomposition can be performed. 
Definition 3.1. Let $X$ and $Y$ be compact subsets of $R^{n}$. We say that $X$ and $Y$ are $M$-regularly situated if for any $h>0$ there exist constants $h^{\prime}>0$ and $C>0$ such that for any $m \in N$,

$$
d(x, X \cap Y)^{p} h^{p} M_{p} / p ! \leq C d(x, Y)^{m} h^{\prime m} M_{m} / m !
$$

for some $p \in N$, for any $x \in X$.

Remark 1. We can use the associated function of $\left(M_{p}\right)$ to write $(3.1)$ as

$$
M^{*}[h d(x, X \cap Y)] \leq C M^{*}\left[h^{\prime} d(x, Y)\right],
$$

where $M^{*}(t)=\exp \left[-M\left(\frac{1}{t}\right)\right]$ and $M(\rho)=\sup _{p} \log \left(\rho^{p} p ! / M_{p}\right)$.

Remark 2. Lojasiewicz [16] has proved that $u \in \mathscr{E}^{\prime}(X \cup Y)$ can be decomposed if and only if either $X \cap Y=\phi$ or there are constants $C>0$ and $\alpha>0$ such that

$$
d(x, Y) \geq C d(x, X \cap Y)^{\alpha} .
$$

Thus we come to know that (3.1) is stronger than (3.2).

Now let $\delta$ be the 'diagonal mapping'

$$
\delta: \mathscr{E}_{M}(X \cup Y) \rightarrow \mathscr{E}_{M}(X) \times \mathscr{E}_{M}(Y)
$$

defined by $\delta(F)=\left(\left.F\right|_{X},\left.F\right|_{Y}\right)$. Then $\delta$ is a continuous injection.

Let $\pi$ be the mapping,

$$
\pi: \mathscr{E}_{M}(X) \times \mathscr{E}_{M}(Y) \rightarrow \mathscr{E}_{M}(X \cap Y)
$$

defined by $\pi(F, G)=\left.F\right|_{X \cap Y}-\left.G\right|_{X \cap Y}$. Then $\pi$ is a continuous surjection by Lemma 2.2 and $\pi \circ \delta=0$.

Now we can obtain the dual mappings,

$$
\delta^{\prime}: \mathscr{E}_{M}^{\prime}(X) \oplus \mathscr{E}_{M}^{\prime}(Y) \rightarrow \mathscr{E}_{M}^{\prime}(X \cup Y)
$$

and

$$
\pi^{\prime}: \mathscr{E}_{M}^{\prime}(X \cap Y) \rightarrow \mathscr{E}_{M}^{\prime}(X) \oplus \mathscr{E}_{M}^{\prime}(Y)
$$

with $\delta^{\prime}(T, S)=T+S$ and $\pi^{\prime}(T)=(T,-T)$.

Theorem 3.2. Under the above hypotheses the following statements are equivalent.

(i) Either $X \cap Y=\phi$ or $X$ and $Y$ are $M$-regularly situated.

(ii) The sequence

$$
0 \rightarrow \mathscr{E}_{M}(X \cup Y) \stackrel{\delta}{\rightarrow} \mathscr{E}_{M}(X) \times \mathscr{E}_{M}(Y) \stackrel{\pi}{\rightarrow} \mathscr{E}_{M}(X \cap Y) \rightarrow 0
$$

is exact.

(iii) The sequence

$$
0 \rightarrow \mathscr{E}_{M}^{\prime}(X \cap Y) \stackrel{\pi^{\prime}}{\rightarrow} \mathscr{\mathscr { E }}_{M}^{\prime}(X) \oplus \mathscr{\mathscr { E }}_{M}^{\prime}(Y) \stackrel{\delta^{\prime}}{\rightarrow} \mathscr{E}_{M}^{\prime}(X \cup Y) \rightarrow 0
$$

is exact.

(iv) Any ultradistribution $u \in \mathscr{E}_{M}^{\prime}(X \cup Y)$ can be decomposed as $u_{1}+u_{2}$ where $u_{1} \in \mathscr{E}_{M}^{\prime}(X)$ and $u_{2} \in \mathscr{E}_{M}^{\prime}(Y)$. 
Proof. The equivalence of (ii), (iii), and (iv) is easily obtained from the theory of duality in DFS-space. Now we will prove the equivalence of (i) and (ii).

The proof of (i) $\Rightarrow$ (ii). We have only to show that $\operatorname{Ker} \pi \subset \operatorname{Im} \delta$.

Let $F=\left(f^{k}\right) \in \mathscr{E}_{M}(X)$ and $G=\left(g^{k}\right) \in \mathscr{E}_{M}(Y)$ with $f^{k}=g^{k}$ on $X \cap Y$ for all $k$. Define a jet $H=\left(H^{k}\right)$ by

$$
H^{k}(x)= \begin{cases}f^{k}(x), & x \in X, \\ g^{k}(x), & x \in Y .\end{cases}
$$

Now we must show that $H$ is a Whitney jet of class $M$ on $X \cup Y$. But $H$ satisfies the derivative condition (2.2). Then to prove the remaining condition (2.3) we show that there are constants $A>0$ and $C>0$ such that

$$
\left|\left(R_{y}^{m} H\right)^{k}(x)\right| \leq \frac{C|x-y|^{m-|k|+1} A^{m+1} M_{m+1}}{(m-|k|+1) !}, \quad|k| \leq m, m \in N .
$$

The case where $x$ and $y$ both belong to $X$ or both belong to $Y$ is clear. Therefore, we may suppose that $x \in X$ and $y \in Y$. But it reduces to the case where $G=0$. In fact, if we extend $G$ to $\tilde{G} \in \mathscr{E}_{M}(X \cup Y)$ and replace $F$ by $F-\tilde{G}$ then we obtain it. Thus (3.3) is reduced to

$$
\left|f^{k}(x)\right| \leq \frac{C|x-y|^{m-|k|+1} A^{m+1} M_{m+1}}{(m-|k|+1) !}, \quad|k| \leq m, m \in N
$$

for all $x \in X$ and $y \in Y$.

Since $F=\left(f^{k}\right)$ is a jet of class $M$ on $X$ there exist $h>0$ and $C>0$ such that

$$
\left|f^{k}(x)\right| \leq \frac{C|x-z|^{m^{\prime}-|k|+1} h^{m^{\prime}+1} M_{m^{\prime}+1}}{(m-|k|+1) !}, \quad|k| \leq m^{\prime}, \quad m^{\prime} \in N
$$

for all $x \in X$ and $z \in X \cap Y$.

From (i) and (2.1) we obtain constants $A^{\prime}>0$ and $C^{\prime}>0$ such that for any $m^{\prime \prime} \in N$,

$$
\frac{|x-z|^{p}(h H)^{p} M_{p}}{p !} \leq \frac{C^{\prime}|x-y|^{m^{\prime \prime}} A^{\prime m^{\prime \prime}} M_{m^{\prime \prime}}}{m^{\prime \prime} !}
$$

for some $p \in N$, for some $z \in X \cap Y$, for $x \in X$ and $y \in Y$.

Let $m$ be an arbitrary integer and $|k| \leq m$. If we take $m^{\prime \prime}=m-|k|+1$ and $m^{\prime}=p+|k|-1$ in (3.5) and (3.6) respectively, it follows that

$$
\begin{aligned}
\left|f^{k}(x)\right| & \leq C|x-z|^{p} h^{p+|k|} M_{p+|k|} / p ! \\
& \leq C|x-z|^{p}(h H)^{p} M_{p}(h H)^{|k|} M_{|k|} / p ! \\
& \leq C C^{\prime}|x-y|^{m-|k|+1} A^{\prime m-|k|+1} M_{m-|k|+1}(h H)^{|k|} M_{|k|} /(m-|k|+1) ! \\
& \leq C^{\prime \prime}|x-y|^{m-|k|+1} A^{\prime m+1} M_{m+1} /(m-|k|+1) !,
\end{aligned}
$$

where $C^{\prime \prime}=C C^{\prime}$ and $A^{\prime \prime}=\max \left\{1, A^{\prime}, h H\right\}$, which is required. 
The proof of (ii) $\Rightarrow$ (i). We assume that $\operatorname{Ker} \pi=\operatorname{Im} \delta$. Then $\operatorname{Im} \delta$ is closed, so the open mapping theorem for DFS-space implies that $\delta$ is a homomorphism. Thus for any $A>0$ and $B>0$ there are constants $A^{\prime}>0$ and $C>0$ such that

$$
\|F\|_{X \cup Y, A^{\prime}} \leq C\left[\|F\|_{X, n A}+\|F\|_{Y, B}\right], \quad F \in \mathscr{E}_{M}(X \cup Y) .
$$

In particular, it is true that for $F=\left(f^{k}\right)$ vanishing on $Y$,

$$
\left|f^{k}(x)\right| \leq C|x-y|^{m-|k|+1} A^{\prime m+1} M_{m+1}\|F\|_{X, n A} /(m-|k|+1) !
$$

for any $m \in N,|k| \leq m$, and $x, y \in X \cup Y$. In view of Lemma 2.2, (3.9) is true for all $F=\left(D^{k} \phi\right), \phi \in C_{M}\left(R^{n}\right)$ whose supports are in $R^{n} \backslash Y$. Then it follows from (2.6) that (3.9) becomes

$$
\left|D^{k} \phi(x)\right| \leq 2 C|x-y|^{m-|k|+1} A^{\prime m+1} M_{m+1}|\phi|_{L, A} /(m-|k|+1) !,
$$

where $L$ is the convex hull of $X$.

Let $\phi_{0}$ be an ultradifferentiable function with $\phi(0)=1$ and supp $\phi_{0} \subset$ $\{x|| x \mid<1\}$ and $\phi(x)=\phi_{0}\left(\left(x-x_{0}\right) / \varepsilon\right)$ where $x_{0} \in X$ and $\varepsilon=d\left(x_{0}, X \cap Y\right)$. Then supp $\phi$ does not meet $Y$. Applying (3.10) to $\phi(x)$ we have

$$
1 \leq 2 C\left|x_{0}-y\right|^{m+1} A^{\prime m+1} M_{m+1}|\phi|_{L, A} /(m+1) ! .
$$

On the other hand, since $|\phi|_{L, A}$ is positive it follows that there exists an $n$-tuple $p \in N^{n}$ such that

$$
\sup _{\substack{x \in L \\ k \in N^{n}}} \frac{\left|D^{k} \phi(x)\right|}{\varepsilon^{|k|} A^{|k|} M_{|k|}} \leq 2 \frac{\left|D^{p} \phi(x)\right|}{\varepsilon^{|p|} A^{|p|} M_{|p|}} .
$$

Combining (3.11) and (3.12) it follows that

$$
d\left(x_{0}, X \cap Y\right)^{|p|} A^{|p|} M_{|p|} / p ! \leq C^{\prime}\left|x_{0}-y\right|^{m+1} A^{\prime m+1} M_{m+1} /(m+1) ! .
$$

Since $x_{0}$ is arbitrary, this implies (3.1).

On the other hand, if $X$ and $Y$ are disjoint then all things considered above are trivial. Therefore, the theorem is proved.

\section{ACKNOWLEDGMENT}

The authors are grateful to A. Kaneko for his advice in preparing this paper.

\section{REFERENCES}

1. G. Björck, Linear partial differential operators and generalized distributions, Ark. Mat. 6 (1965), 351-407.

2. J. Boman, On the intersection of classes of infinitely differentiable functions, Ark. Mat. 5 (1964), 301-309.

3. J. Bruna, An extension theorem of Whitney type for non-quasianalytic class of functions, J. London Math. Soc. (2) 22 (1980), 495-505.

4. J. Chazarain, Problèmes des Cauchy dans espaces d'ultradistributions, C. R. Acad. Sci. Paris 266 (1968), 10-13. 
5. S. Y. Chung, Ultradifferentiable mappings and decomposition of ultradistributions, $\mathrm{Ph}$. D. Dissertation, Seoul National University, 1988.

6. P. Cohen, A simple proof of the Denjoy-Carleman theorem, Amer. Math. Monthly 75 (1968), 26-31.

7. J. Dieudonné and L Schwartz, La dualité dans les espaces $(F)$ et $(L F)$, Ann. Inst. Fourier 1 (1950), 61-101.

8. J. L. Ermine, Développements asymptotiques et microfonctions dans les classes de Gevrey, Séminaire Goulaouic-Meyer-Schwartz, 1981-1982.

9. A. Grothendieck, Sur les espaces $(F)$ et (DF), Summa Brasil. Math. 3 (1954), 57-122.

10. __ Topological vector spaces, Gordon and Breach, New York, 1973.

11. L. Hörmander, Between distributions and hyperfunctions, Astérisque 131 (1985), 89-106.

12. __ The analysis of linear partial differential operator I, Springer-Verlag, New York, 1983.

13. H. Komatsu, Projective and injective limits of weakly compact sequences of locally convex spaces, J. Math. Soc. Japan 19 (1967), 366-383.

14. __ Ultradistributions I, structure theorem and a characterization, J. Fac. Sci. Univ. Tokyo Sect IA 20 (1970), 25-105.

15. J. L. Lions and E. Magenes, Espaces de fonctions et distributions du type de Gevrey et problème aux limites paraboliques, Ann. Mat. Pure Appl. Ser. 4, 68 (1965), 341-417.

16. S. Lojasiewicz, Sur le problème de la division, Studia Math. 8 (1959), 87-136.

17. B. Malgrange, Ideals of differentiable functions, Tata Institute, Bombay and Oxford University Press, 1966.

18. C. Roumieu, Sur quelques extensions de la notion de distribution, Ann. Sci. École Norm. Sup. (4) 77 (1960), 41-121.

19. _ _ Ultra-distribution définis sur $R^{n}$ et sur certaines classes de variétés differentiables, $\mathrm{J}$. Analyse Math. 10 (1962), 153-192.

Department of Mathematics, Duksung Women's University, Seoul, Korea 132-031

Department of Mathematics, Seoul National University, Seoul, Korea 151-742 